University of South Carolina

Scholar Commons

2005

\title{
Interphase Exchange Coupling in Fe/Sm-Co Bilayers with Gradient Fe Thickness
}

\author{
Ming-Hui Yu \\ Jason R. Hattrick-Simpers \\ University of South Carolina - Columbia, simpers@cec.sc.edu \\ Ichiro Takeuchi \\ Jing Li
}

Z. L. Wang

See next page for additional authors

Follow this and additional works at: https://scholarcommons.sc.edu/eche_facpub

Part of the Electromagnetics and Photonics Commons, Optics Commons, and the Other Chemical Engineering Commons

\footnotetext{
Publication Info

Published in Journal of Applied Physics, Volume 98, Issue 6, 2005, pages \#063908-.

(C) Journal of Applied Physics 2005, AIP (American Institute of Physics).

Yu, M-H., Hattrick-Simpers, J., Takeuchi, I., Li, J., Wang, Z. L., Liu, J. P., Lofland, S. E., Tyagi, S., Freeland, J. W., Giubertoni, D., Bersani, M., \& Anderle, M. (15 September 2005). Interphase Exchange Coupling in Fe/ Sm-Co Bilayers with Gradient Fe Thickness. Journal of Applied Physics, 98 (6), \#063908.

http://dx.doi.org/10.1063/1.2042529
}

This Article is brought to you by the Chemical Engineering, Department of at Scholar Commons. It has been accepted for inclusion in Faculty Publications by an authorized administrator of Scholar Commons. For more information, please contact digres@mailbox.sc.edu. 


\section{Author(s)}

Ming-Hui Yu, Jason R. Hattrick-Simpers, Ichiro Takeuchi, Jing Li, Z. L. Wang, J. P. Liu, S. E. Lofland, Somdev Tyagi, J. W. Freeland, D. Giubertoni, M. Bersani, and M. Anderle 


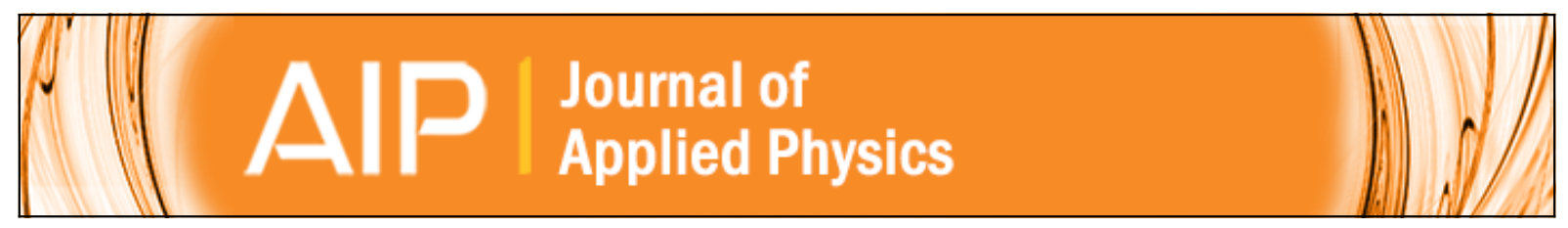

\section{Interphase exchange coupling in $\mathrm{Fe} / \mathrm{Sm}$ - Co bilayers with gradient Fe thickness}

Ming-hui Yu, Jason Hattrick-Simpers, Ichiro Takeuchi, Jing Li, Z. L. Wang, J. P. Liu, S. E. Lofland, Somdev Tyagi , J. W. Freeland, D. Giubertoni, M. Bersani, and M. Anderle

Citation: Journal of Applied Physics 98, 063908 (2005); doi: 10.1063/1.2042529

View online: http://dx.doi.org/10.1063/1.2042529

View Table of Contents: http://scitation.aip.org/content/aip/journal/jap/98/6?ver=pdfcov

Published by the AIP Publishing

\section{Articles you may be interested in}

Soft x-ray magnetic circular dichroism study of $\mathrm{Mn}-\mathrm{Ir} / \mathrm{Co}$ - Fe bilayers with giant exchange anisotropy Appl. Phys. Lett. 89, 172501 (2006); 10.1063/1.2364116

Recoil hysteresis of $\mathrm{Sm}-\mathrm{Co} / \mathrm{Fe}$ exchange-spring bilayers

J. Appl. Phys. 98, 113906 (2005); 10.1063/1.2138785

Reversible magnetization processes and energy density product in $\mathrm{Sm}-\mathrm{CoFe}$ and $\mathrm{Sm}-\mathrm{Co} / \mathrm{Co}$ bilayers J. Appl. Phys. 93, 6489 (2003); 10.1063/1.1558245

Exchange coupling in epitaxial Sm-Co (1100)/ Nd-Co exchange-spring bilayers J. Appl. Phys. 93, 8122 (2003); 10.1063/1.1538180

Kerr observations of asymmetric magnetization reversal processes in CoFe/lrMn bilayer systems J. Appl. Phys. 93, 5491 (2003); 10.1063/1.1562732

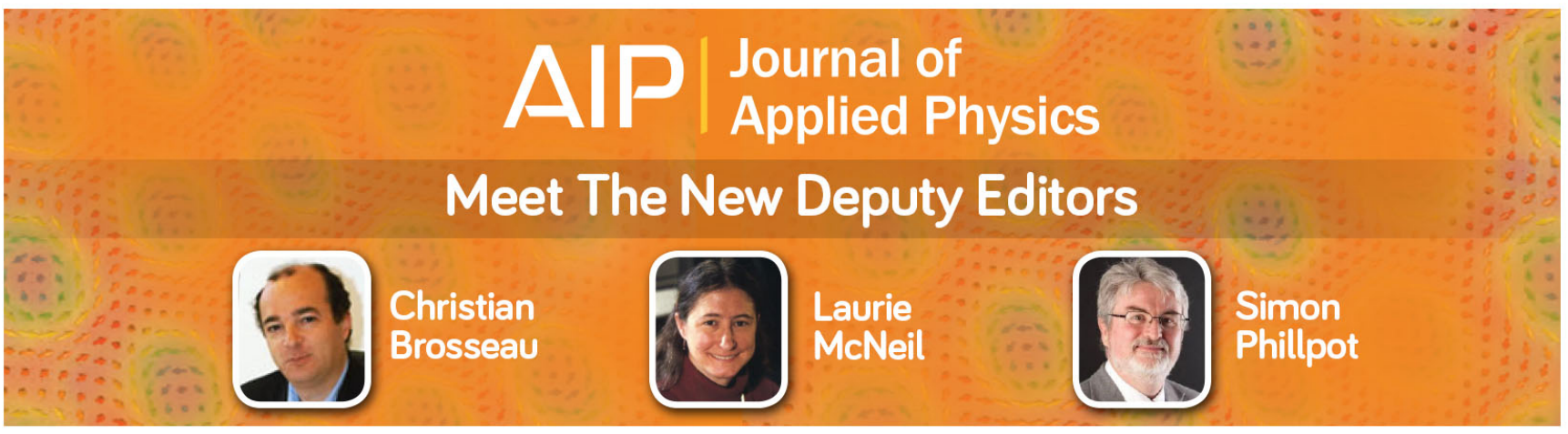




\title{
Interphase exchange coupling in Fe/Sm-Co bilayers with gradient Fe thickness
}

\author{
Ming-hui $\mathrm{Yu}_{\text {, }}^{\text {a) }}$ Jason Hattrick-Simpers, and Ichiro Takeuchi \\ Department of Materials Science and Engineering, University of Maryland, College Park, \\ Maryland 20742 and Center for Superconductivity Research, University of Maryland, College Park, \\ Maryland 20742-4111
}

Jing Li and Z. L. Wang

School of Materials Science and Engineering, Georgia Institute of Technology, Atlanta, Georgia 30332

J. P. Liu

Department of Physics, University of Texas at Arlington, Arlington, Texas 76019

S. E. Lofland and Somdev Tyagi ${ }^{\text {b) }}$

Department of Physics, Rowan University, Glassboro, New Jersey 08028

\section{J. W. Freeland}

Advanced Photon Source, Argonne National Laboratory, Argonne, Illinois 60439

D. Giubertoni, M. Bersani, and M. Anderle

Instituto Trentino di Cultura-II Centro per la Ricerca Scientifica e Tecnologica (ITC-IRST), 38050 Povo-Trento, Italy

(Received 18 May 2005; accepted 4 August 2005; published online 30 September 2005)

We have fabricated $\mathrm{Fe} / \mathrm{Sm}$-Co bilayers with gradient $\mathrm{Fe}$ thicknesses in order to systematically study the dependence of exchange coupling on the thickness of the Fe layer. The Fe layer was deposited at two different temperatures $\left(150\right.$ and $\left.300^{\circ} \mathrm{C}\right)$ to study the effect of deposition temperature on the exchange coupling. Magneto-optical Kerr effect and x-ray magnetic circular dichroism (XMCD) have been employed as nondestructive rapid characterization tools to map the magnetic properties of the gradient samples. Systematic enhancement in exchange coupling between the soft layer and the hard layer is observed as the soft layer thickness is decreased. Separate exchange couplings of the Fe layer with $\mathrm{Co}$ and $\mathrm{Sm}$ in the hard layer are revealed through measuring the element-specific hysteresis curves using XMCD. The single-phase-like magnetization reversal critical thickness increases from $12 \mathrm{~nm}$ for Fe deposited at $150{ }^{\circ} \mathrm{C}$ to $24 \mathrm{~nm}$ for Fe deposited at $300{ }^{\circ} \mathrm{C}$, indicating an important role of the state of the interface in the exchange coupling. (C) 2005 American Institute of Physics. [DOI: 10.1063/1.2042529]

\section{INTRODUCTION}

Enhanced remanence and increased maximum energy product can be achieved in nanocomposite exchange-coupled soft/hard magnet systems by combining high magnetization in the soft magnet with high anisotropy in the hard magnet. ${ }^{1}$ The exchange coupling beneficial to the maximum energy product takes place only if the dimension of the soft phase is smaller than a critical thickness. When the soft-phase dimension is larger than this length, the exchange coupling between the hard and soft phases can no longer fully control the reversal of the soft-phase moment, and the hysteresis loop displays a two-phase-like characteristic (with a step in the loop). Early theoretical calculations had predicted that the critical thickness is twice the domain wall thickness in the hard phase and is not related with the soft phase. ${ }^{2}$ However, recent theoretical and experimental investigations have

\footnotetext{
${ }^{\text {a) }}$ Author to whom correspondence should be addressed; also at Department of Physics, University of Texas at Arlington, Arlington, Texas 76019; FAX (504) 280-3185; electronic mail: myul@uno.edu

b) Also at Department of Physics, Drexel University, Philadelphia, Pennsylvania 19014
}

shown that the critical thickness is also strongly dependent on the soft-phase parameters, ${ }^{3,4}$ including magnetization and anisotropy. Another unresolved aspect of exchange coupling is how the state of the interface influences the exchange coupling. Until very recently, not much attention was paid to the interface effect. ${ }^{5}$ In order to gain clear understanding of the effect of various soft-phase parameters and the interface state on exchange coupling, many combinations of hard-soft composite systems need to be studied. The delicate nature of these effects requires such experiments to be performed under strictly the same conditions varying only one parameter at a time, because subtle run to run variation in conditions can lead to a significant deviation from the expected behavior. This problem can be circumvented by employing the high-throughput synthesis and measurement techniques, where many samples can be studied at a time to minimize the error from condition variation and drastically increase the experimental efficiency. ${ }^{6}$

In this work, combinatorial magnetron sputtering was used to synthesize exchange-coupled magnetic bilayers, where the natural thickness gradient in the deposition geom- 
etry was used to create a wedge thickness profile in the soft layer in order to systematically investigate the thickness dependence of exchange coupling.

\section{EXPERIMENT}

$\mathrm{Fe} / \mathrm{Sm}-\mathrm{Co}$ magnetic bilayers were deposited on $\mathrm{Si}$ (100) substrates in the high-vacuum magnetron sputtering chamber equipped with three parallel sputtering guns (with the base pressure in the $10^{-9}$-Torr range). ${ }^{7} \mathrm{SmCo}_{5}$ and $\mathrm{Fe}$ targets were dc sputtered with an Ar pressure of 5 mTorr. The sputtering power and the target-to-substrate distance were adjusted to optimize the saturation magnetization and coercivity of the Sm-Co layer and the thickness gradient of the Fe layer. The Sm-Co layer was found to be textured with $c$ axis in the film plane. Wavelength dispersive X-ray spectroscopy (WDS) indicated that the composition of the deposited $\mathrm{Sm}-\mathrm{Co}$ layer was $\mathrm{SmCo}_{6.5}$. A uniform thickness of the $\mathrm{Sm}-\mathrm{Co}$ layer with homogeneous magnetic properties was attained in a relatively large region $\left(\sim 9 \times 50 \mathrm{~mm}^{2}\right)$ on the substrate when it was deposited at $550{ }^{\circ} \mathrm{C}$. An Fe layer with a natural thickness gradient of $\sim 0.55 \mathrm{~nm} / \mathrm{mm}$ was formed in the same region of the substrate (inset of Fig. 1). The thickness gradient of the $\mathrm{Fe}$ layer was confirmed by secondaryion-mass spectroscopy (SIMS).

\section{RESULTS AND DISCUSSION}

We have found that depending on the thermal treatment, Sm-Co films can display very different values of coercivity and saturation magnetization. We have used 130-nm-thick $\mathrm{Sm}-\mathrm{Co}$ layers with the saturation magnetization of $530 \mathrm{emu} / \mathrm{cc}$ and the coercivity of $14 \mathrm{kOe}$. This value of saturation magnetization is comparable to that of epitaxial Sm-Co films grown at $600{ }^{\circ} \mathrm{C}$ on Cr-buffered $\mathrm{MgO}(100){ }^{8}$ Subsequently, the $\mathrm{Fe}$ layer was deposited on top of the $\mathrm{Sm}-\mathrm{Co}$ layer after the wafer is cooled to 300 or $150{ }^{\circ} \mathrm{C}$. Afterwards, a $\mathrm{Cr}$ layer $(7 \mathrm{~nm})$ was deposited at room temperature as a capping layer to prevent oxidation of the bilayers.

We have used the magneto-optic Kerr effect (MOKE) and the x-ray magnetic circular dichroism (XMCD) for rapid characterization and mapping of magnetic properties of the thickness gradient bilayer samples. The longitudinal MOKE magnetization loops measured at different spots on the samples using a 633-nm wavelength laser are shown in Fig. 1 [Figs. 1(a) and 1(b) are from bilayers with Fe deposited at 150 and $300{ }^{\circ} \mathrm{C}$, respectively]. Because the penetration depth of the laser is limited to $\sim 20 \mathrm{~nm}$, the MOKE signal is surface sensitive and mainly reveals the magnetic behavior of the upper soft layer in the magnetic bilayers. The field dependence of the Kerr effect represents the magnetization process of the soft layer due to the applied field. ${ }^{9}$ Enhancement in the exchange coupling between the soft layer and the hard layer with the decreased soft layer thickness is clearly evident, as minor loops get smaller and it becomes harder to reverse the polarization of the soft layer when the Fe layer gets thinner. The maximum applied field for this measurement was $10 \mathrm{kOe}$, which is not large enough to saturate the Fe layer when it is strongly coupled to the hard Sm-Co layer.
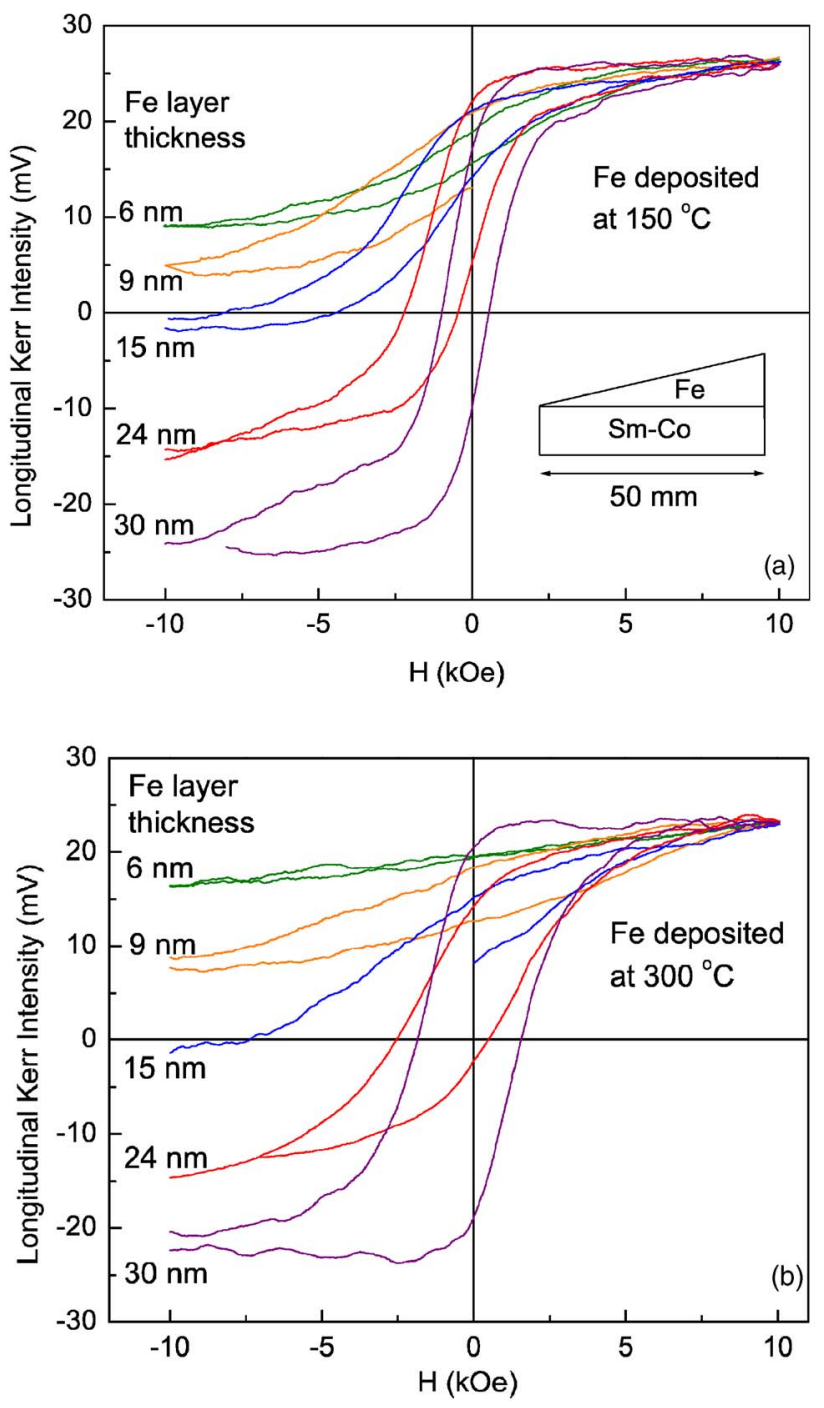

FIG. 1. (Color online) Longitudinal Kerr magnetization loops taken at different spots on $\mathrm{Fe} / \mathrm{Sm}-\mathrm{Co}$ bilayers with gradient $\mathrm{Fe}$ thickness deposited at $150{ }^{\circ} \mathrm{C}$ (a) and $300{ }^{\circ} \mathrm{C}$ (b). The inset of (a) shows the schematic of the samples.

Therefore, most of the loops we obtained are minor loops and asymmetric. Comparing Fig. 1(a) with Fig. 1(b), we conclude that it is harder to reverse the magnetization of the $\mathrm{Fe}$ layer deposited at $300{ }^{\circ} \mathrm{C}$, indicating stronger coupling. The coercivity of the $30-\mathrm{nm}$ Fe layer in Fig. 1(b) is larger than that in Fig. 1(a).

In order to probe the magnetic property of the buried hard layer, we have performed XMCD utilizing a synchrotron $\mathrm{x}$-ray beam. Through the use of circularly polarized $\mathrm{x}$ rays tuned to an elemental absorption resonance, we can probe the individual magnetic behavior of soft and hard layers separately. The XMCD signal represents the difference between the absorption of left and right circularly polarized $\mathrm{x}$ rays, which effectively measures the populations of the up and down spin orientations. ${ }^{10}$ XMCD measurements were performed at beamline 4-ID-C of Advanced Photon Source using a 7-T superconducting magnet. ${ }^{11}$ The fluorescence yields at the Fe $L_{3}$, Co $L_{2}$, and $\mathrm{Sm} M_{5}$ edges were used to record the element-specific hysteresis curves at $100 \mathrm{~K}$ at various spots on the samples (Fig. 2). Only half loops were 
Fe at $150^{\circ} \mathrm{C}$

Fe at $300^{\circ} \mathrm{C}$

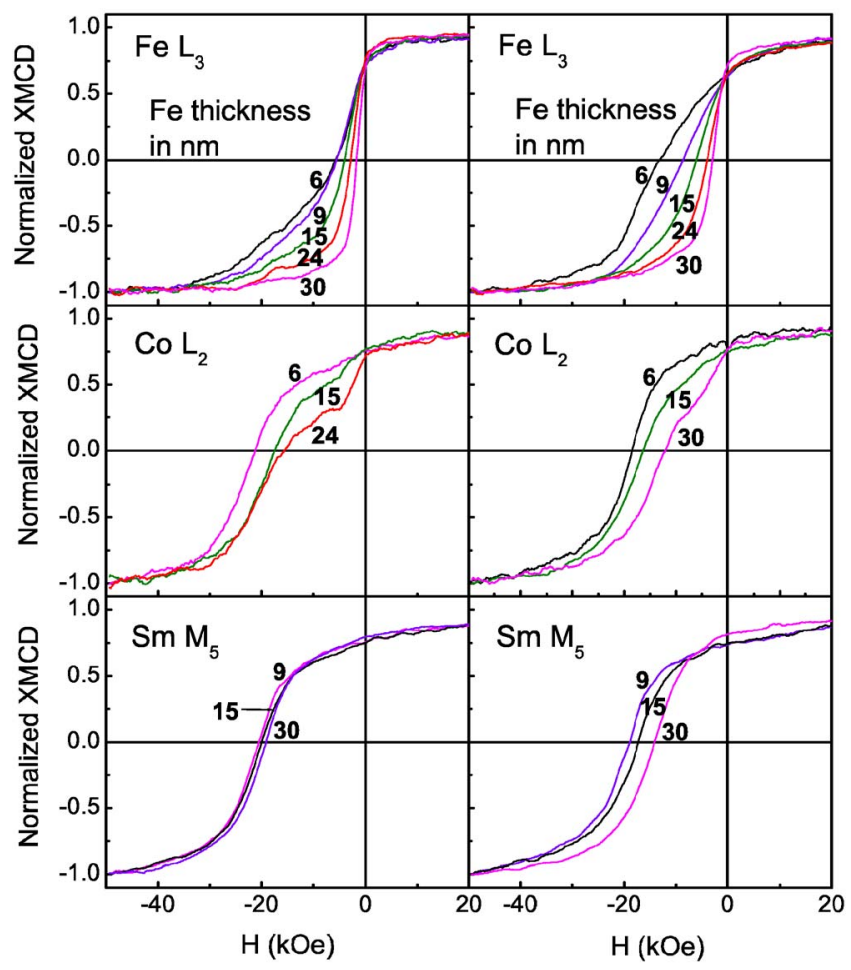

FIG. 2. (Color online) Fe $L_{3}$, Co $L_{2}$, and $\mathrm{Sm} M_{5}$ edge XMCD hysteresis curves taken at different spots on $\mathrm{Fe} / \mathrm{Sm}-\mathrm{Co}$ bilayers with gradient $\mathrm{Fe}$ thickness deposited at $150{ }^{\circ} \mathrm{C}$ (left) and $300{ }^{\circ} \mathrm{C}$ (right). The number on the curve is the Fe thickness in the unit of nanometers.

recorded to make efficient use of the beam time. It is interesting to note that the shape of the hysteresis curves and the coercivity are different for Co and $\mathrm{Sm}$. This indicates that there is a field-induced noncollinear spin configuration between the Co and Sm magnetic moments. In some twosublattice ferromagnets, the strong competition between the exchange interaction, magnetocrystalline anisotropies, and Zeeman energy could produce the field-induced noncollinear spin configurations. ${ }^{12}$ In our case, we found that the noncollinear spin configuration is not present in a single $\mathrm{Sm}-\mathrm{Co}$ layer sample (not shown here), which indicates that the noncollinear spin configuration is not a bulk effect and that it is closely related with the difference in interlayer $\mathrm{Co}-\mathrm{Fe}$ and $\mathrm{Sm}-\mathrm{Fe}$ exchange interactions. Because $\mathrm{Sm} 4 f$ electrons are screened by the outer-shell electrons, they are subjected to stronger interaction with the crystal field, and weaker interaction with $\mathrm{Fe}$ atoms, compared to $3 d$ electrons in Co atom. The stronger Fe thickness dependence of the Sm coercivity for $300{ }^{\circ} \mathrm{C}$-deposited $\mathrm{Fe}$ indicates stronger exchange coupling between $\mathrm{Sm}$ and $\mathrm{Fe}$ atoms. It is worthwhile to note that the Co hysteresis curves show a knee when the soft layer is thicker than 15 and $30 \mathrm{~nm}$ for the Fe layer deposited at 150 and $300{ }^{\circ} \mathrm{C}$, respectively. This might be due to the coexistence of multiphases in the Sm-Co layer or the presence of composition inhomogeneity in the film. Indeed, $\mathrm{SmCo}_{5}$, rhombohedral $\mathrm{Sm}_{2} \mathrm{Co}_{17}$, and hexagonal $\mathrm{SmCo}_{8.5}$ were observed in the film by high-resolution transmission electron microscopy (HRTEM). ${ }^{13}$ It should be noted that the microscopic composition inhomogeneity observed in the nanom-

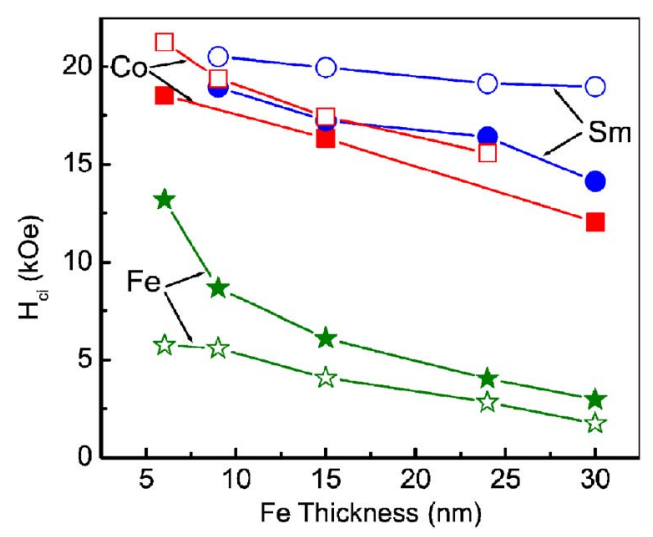

FIG. 3. (Color online) Fe thickness dependence of element-specific coercivity for $\mathrm{Fe}, \mathrm{Co}$, and $\mathrm{Sm}$ deduced from $\mathrm{XMCD}$ data for $\mathrm{Fe}$ deposited at $150{ }^{\circ} \mathrm{C}$ (open) and $300{ }^{\circ} \mathrm{C}$ (solid).

eter region is not in conflict with the above-mentioned macroscopic magnetic property homogeneity observed on the millimeter scale in the Sm-Co layer. Different $\mathrm{Sm}-\mathrm{Co}$ phases and compositions can have different magnetocrystalline anisotropies. They can also have different critical thicknesses with the Fe layer, which give rise to the knee when the Fe thickness is larger than the smallest critical thickness. The stronger exchange coupling with the higher-temperature deposited Fe seems to increase the smallest critical thickness. No obvious knee can be observed in the Sm hysteresis curves. This indicates that the exchange coupling between $\mathrm{Sm}$ and $\mathrm{Fe}$ is probably not sensitive to the phase and composition variations.

The Fe thickness dependence of the element-resolved coercivities as deduced from the XMCD result is plotted in Fig. 3. The coercivity of the Fe layer in the exchangecoupled bilayer can become much larger than that of an independently measured single Fe layer $(\sim 500 \mathrm{Oe})$, and it increases with the decreased Fe layer thickness. The larger Fe coercivity, the better squareness, and the stronger thickness dependence in the higher-temperature deposited Fe bilayer indicate the more favorable exchange coupling, which is consistent with the results obtained from MOKE.

In order to confirm the results, we have also performed $M-H$ loop measurements using a superconducting quantum interface device (SQUID) magnetometer on selected regions of the samples by cutting the bilayer wafer into $5 \times 5$ - $\mathrm{mm}^{2}$-sized squares (Fig. 4). As the Fe thickness is increased, the hysteresis curves changes from the single-phaselike magnetization reversal to the two-phase magnetization reversal. The single-phase-like magnetization reversal can be sustained up to $24 \mathrm{~nm}$ for $\mathrm{Fe}$ deposited at $300{ }^{\circ} \mathrm{C}$, but only $12 \mathrm{~nm}$ for Fe deposited at $150{ }^{\circ} \mathrm{C}$.

We believe that the observed enhanced coupling for bilayers with Fe deposited at the higher temperature is due to the interdiffusion of the $\mathrm{Fe}$ atoms and concomitant formation of a coherent interface. SIMS analyses of the interfaces indicate that in comparison to Fe deposited at $150{ }^{\circ} \mathrm{C}, 300{ }^{\circ} \mathrm{C}$ deposition results in an interface region approximately $6 \mathrm{~nm}$ where Fe had interdiffused. It is interesting to note that a separate experiment performed with $\mathrm{Co} / \mathrm{Sm}$-Co bilayers did 

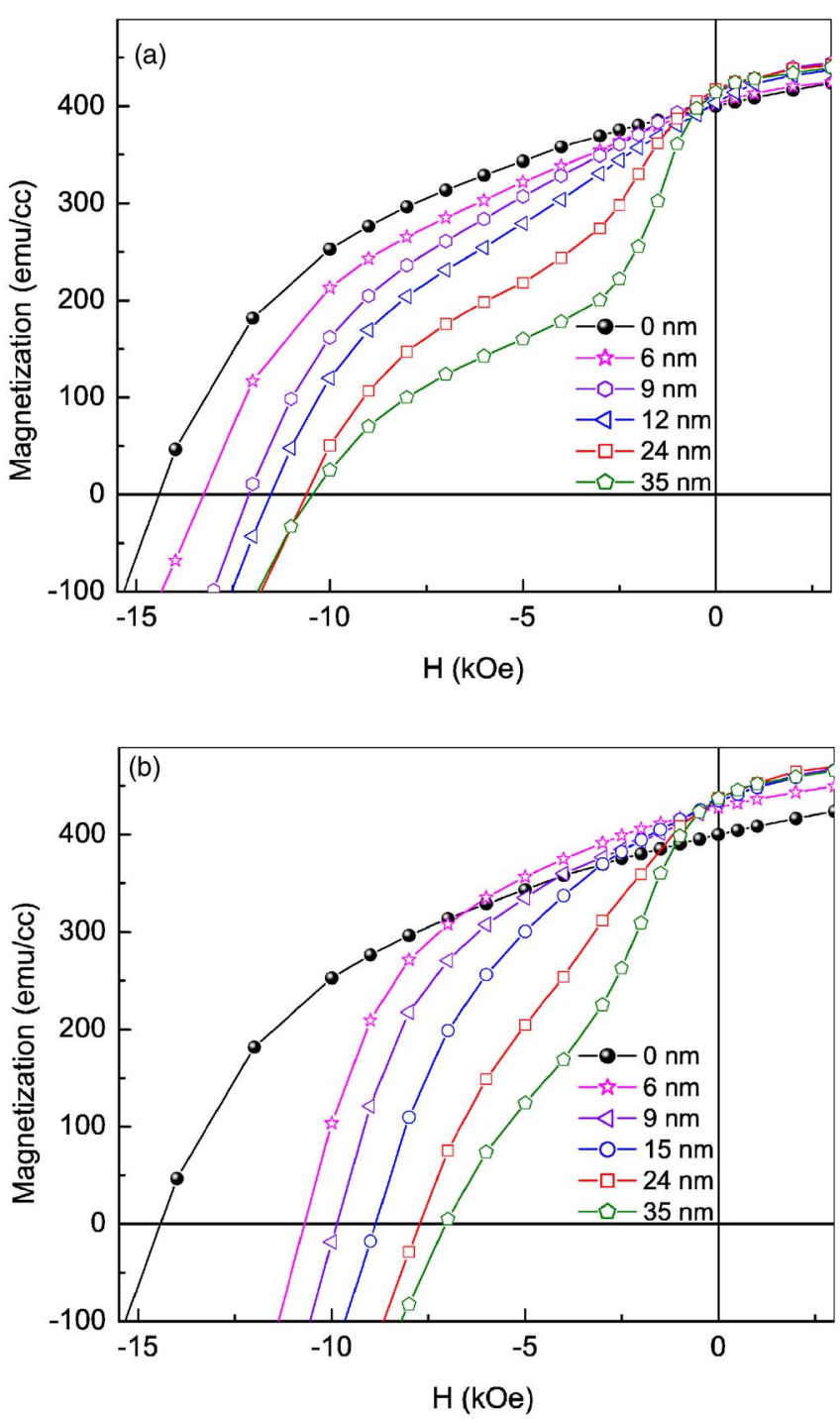

FIG. 4. (Color online) Room-temperature hysteresis curves for $\mathrm{Fe} / \mathrm{Sm}-\mathrm{Co}$ bilayers with different $\mathrm{Fe}$ thickness deposited at $150{ }^{\circ} \mathrm{C}$ (a) and $300{ }^{\circ} \mathrm{C}$ (b)

not exhibit any interdiffusion for the Co deposited at $300{ }^{\circ} \mathrm{C}$ indicating that the interdiffusion in the $\mathrm{Fe} / \mathrm{Sm}-\mathrm{Co}$ case is most likely between $\mathrm{Fe}$ and $\mathrm{Co}$ atoms.

Figure 5 is a HRTEM image of the $\mathrm{Fe} / \mathrm{Sm}-\mathrm{Co}$ bilayer

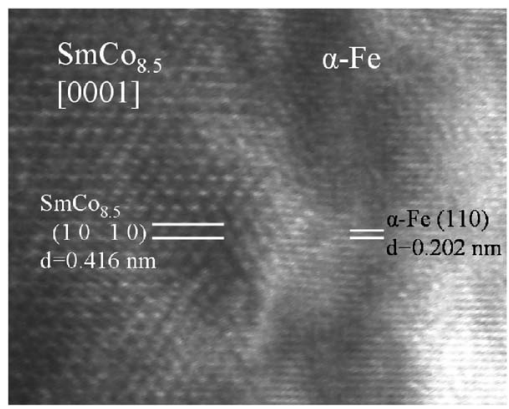

FIG. 5. HRTEM of the $\mathrm{Fe}(110) / / \mathrm{SmCo}_{8.5}(10-10)$ interface (Fe deposited at $\left.300{ }^{\circ} \mathrm{C}\right)$. A coherent interface was observed. with $\mathrm{Fe}$ deposited at $300{ }^{\circ} \mathrm{C}$ displaying an epitaxial coherent interface that had formed between the layers. Such an interface was not observed for the bilayer with Fe deposited at $150{ }^{\circ} \mathrm{C}$, and therefore we directly associate the coherent interface as the cause of the enhanced exchange coupling. The formation of the coherent interface was likely facilitated by the interdiffusion of the $\mathrm{Fe}$ and $\mathrm{Co}$ at the higher temperature. Thus, the interface is perhaps best described as actually between $\mathrm{Fe}-\mathrm{Co} / \mathrm{Sm}-\mathrm{Fe}-\mathrm{Co} .{ }^{14}$ We believe that it is the combination of the interdiffused region with gradual composition change and the reduced density of defects (as evidenced by the lattice coherence) which has given rise to an enhanced interphase exchange coupling.

\section{CONCLUSIONS}

In conclusion, we have demonstrated the utility of highthroughput studies in investigation of exchange coupling in the gradient thickness $\mathrm{Fe} / \mathrm{Sm}-\mathrm{Co}$ bilayers. MOKE and $\mathrm{XMCD}$ have been employed to rapidly characterize the interlayer exchange coupling. The field-induced noncollinear configuration between the Co and Sm magnetic moments and the different $\mathrm{Sm}-\mathrm{Fe}$ and $\mathrm{Co}-\mathrm{Fe}$ exchange couplings were revealed by measuring the element-specific hysteresis curves using XMCD. Deposition of the soft layer at $300{ }^{\circ} \mathrm{C}$ was found to enhance the interphase exchange coupling due to the formation of an intermixing boundary with a lattice coherent interface.

\section{ACKNOWLEDGMENTS}

This work was supported by DARPA through ARO under Grant No. DAAD19-03-1-0038, NSF DMR 0231291, and NSF DMR 00-80008, ONR N00014-05-1-0497 (MURI). It was also partially funded by the Provincia Autonoma di Trento, Italy, under the Microcombi project.

${ }^{1}$ E. F. Kneller and R. Hawig, IEEE Trans. Magn. 27, 3588 (1991).

${ }^{2}$ T. Schrefl, H. Kronmüller, and J. Fidler, J. Magn. Magn. Mater. 127, L273 (1993).

${ }^{3}$ Z. S. Shan, J. P. Liu, V. M. Chakka, H. Zeng, and J. S. Jiang, IEEE Trans. Magn. 38, 2907 (2002).

${ }^{4}$ Z. J. Guo, J. S. Jiang, J. E. Pearson, S. D. Bader, and J. P. Liu, Appl. Phys. Lett. 81, 2029 (2002).

${ }^{5}$ J. S. Jiang et al., Appl. Phys. Lett. 85, 5293 (2004).

${ }^{6}$ H. Koinuma and I. Takeuchi, Nat. Mater. 3, 429 (2004).

${ }^{7}$ I. Takeuchi et al., Nat. Mater. 2, 180 (2003).

${ }^{8}$ E. E. Fullerton, J. S. Jiang, C. H. Sowers, J. E. Pearson, and S. D. Bader, Appl. Phys. Lett. 72, 380 (1998).

${ }^{9}$ O. Hellwig, J. B. Kortright, K. Takano, and E. E. Fullerton, Phys. Rev. B 62, 11694 (2000).

${ }^{10}$ C. T. Chen et al., Phys. Rev. Lett. 75, 152 (1995).

${ }^{11}$ J. W. Freeland, J. C. Lang, G. Srajer, R. Winarski, D. Shu, and D. M. Mills, Rev. Sci. Instrum. 73, 1408 (2002).

${ }^{12}$ Z.-D. Zhang, T. Zhao, X. K. Sun, and Y. C. Chuang, J. Appl. Phys. 71, 3434 (1992).

${ }^{13}$ A detailed HRTEM structural characterization for the $\mathrm{Fe} / \mathrm{Sm}-\mathrm{Co}$ magnetic bilayer will be published separately.

${ }^{14}$ Further high-resolution interface studies using energy dispersive $\mathrm{x}$-ray analysis (EDAX) and electron-energy-loss spectroscopy (EELS) are currently underway. 